\title{
Snake instability of dark solitons across the BEC-BCS crossover: An effective-field-theory perspective
}

\author{
G. Lombardi, ${ }^{1, *}$ W. Van Alphen, ${ }^{1, \dagger}$ S. N. Klimin, ${ }^{1,2}$ and J. Tempere ${ }^{1,3}$ \\ ${ }^{1}$ TQC, Universiteit Antwerpen, Universiteitsplein 1, 2610 Antwerpen, Belgium \\ ${ }^{2}$ Department of Theoretical Physics, State University of Moldova, 2009 Chișinău, Moldova \\ ${ }^{3}$ Lyman Laboratory of Physics, Harvard University, Cambridge, Massachusetts 02138, USA
}

(Received 25 November 2016; published 7 September 2017)

\begin{abstract}
In the present article the snake instability mechanism for dark solitons in superfluid Fermi gases is studied in the context of a recently developed effective field theory [S. N. Klimin et al., Eur. Phys. J. B 88, 122 (2015)]. This theoretical treatment has proven to be suitable to study stable dark solitons in quasi-one-dimensional setups across the BEC-BCS crossover. In this paper the nodal plane of the stable soliton solution is perturbed by adding a transverse modulation. The numerical solution of the system of coupled nonlinear differential equations describing the amplitude of the perturbation leads to an estimate of the growth rate and characteristic length scale of the instability, which are calculated for a wide range of interaction regimes and compared to other theoretical predictions. The behavior of the maximum transverse size that the atomic cloud can have in order to preserve the stability is described across the BEC-BCS crossover. The analysis of the effects of spin imbalance on this critical length reveals a stabilization of the soliton with increasing imbalance and therefore provides the experimental community with a method to achieve the realization of stable solitons in real three-dimensional configurations, without reducing the system dimensionality.
\end{abstract}

DOI: 10.1103/PhysRevA.96.033609

\section{INTRODUCTION}

Solitons are solitary waves that constitute the solution of a wide range of differential equations involving an interplay between nonlinearity and dispersive effects. They have been examined in many physical systems including optics, fluid mechanics, plasmas, and ultracold gases. In recent years, dark solitons were experimentally observed in both BoseEinstein condensates [1-4] and Fermi superfluids, where the experimental realization proved to be less straightforward: Initially [5], solitonlike defects were observed, but their long lifetime and high effective mass lead to the conclusion [6] that what had been detected were in fact solitonic vortices, the product of the decay of a planar soliton. Finally, in [7] the entire evolution and decay of a dark soliton were experimentally observed.

In ultracold quantum systems dark solitons manifest themselves as localized density dips propagating at constant velocity on a stable background. In correspondence with these defects, the order parameter shows a dip in the amplitude profile and a jump in the phase profile.

From a theoretical point of view, dark solitons were widely examined in both BECs [8,9] and Fermi superfluids [10-14]. As concerns the stability of the soliton, it was demonstrated [15-17] that, while a soliton in one-dimensional (1D) configurations is stable, the presence of a transverse dimension provokes its decay through the snake instability mechanism. The name snake instability [18] comes from the consideration that in the decay process the depletion plane starts to oscillate until the defect loses its soliton character: The cascade of solitonic excitations arising from the snaking of a planar soliton was observed experimentally

\footnotetext{
*giovanni.lombardi@uantwerpen.be

†wout.vanalphen@uantwerpen.be
}

both in BECs [19] and in Fermi superfluids [7]. Stable solitons were predicted in BECs subject to an external potential [20] and in dipolar BECs [21,22]. In fermionic systems, theorists have analyzed the snake instability mechanism by employing different methods, e.g., the hydrodynamic approximation, the random-phase approximation (RPA) approach, the numerical solution of the time-dependent Bogoliubov-de Gennes (BdG) equations [23], and calculations [24] based on a coarse-grained version of the BdG equations introduced in [25].

In the present paper we study the snake instability by using a recently developed effective-field theory (EFT) [26] capable of describing Fermi superfluids across the BEC-BCS crossover regime in a wide temperature domain. This theory is based on the assumption that the order parameter changes slowly in both space and time, corresponding to the condition [27] that the pair field should vary over a spatial region much larger than the pair correlation length [28,29] (also referred to as the Pippard length in the context of superconductors). The consequent limitations and validity domain of the EFT have been discussed in detail in [30]. The theory has already been employed in the description of the stable soliton solution in (quasi-)1D setups in different regimes of temperature and imbalance $[30,31]$. One goal of our work is to analyze the spectrum of the instability and to compare the results of the EFT with those of other theoretical approaches across the BEC-BCS crossover regime. The snake instability is a long-wavelength phenomenon: The inverse of the maximum wave number $k_{c}$ for which the soliton is unstable, i.e., $k_{c}^{-1}$, can therefore give an estimate of the minimum transverse length that the atomic cloud must have in order for the decay to occur in experiment. Since in experiments with Fermi superfluids no stable solitons have been observed yet, the estimate of this quantity can help design future experimental observations. The study carried out in this work is based on the perturbation of stationary soliton solutions obtained from the analytic expressions for the phase and amplitude profiles of the order 
parameter derived in [30,31], which are valid for a uniform system. The recent realization of boxlike optical traps [32] provides the opportunity to test the predictions of the present work in experiment.

The high experimental control obtained in ultracold fermionic systems enables one to tune a broad variety of parameters, from temperature to interaction strength. In addition, physicists managed to experimentally engineer spinimbalanced systems $[33,34]$, in which the populations of particles with spin up and spin down are uneven. From a theoretical point of view, the difference in the spin populations has been demonstrated to strongly affect the pairing mechanism [35]. In this work the effects of imbalance are analyzed with respect to the minimal transverse length $k_{c}^{-1}$ and the stability of the stationary soliton solution.

\section{PERTURBATIVE TREATMENT}

The system under consideration is an ultracold Fermi gas in which particles in states with opposite pseudospin interact via an $s$-wave contact potential. In the context of a recently developed effective-field theory [26], this system can be described across the BEC-BCS crossover regime in terms of the superfluid order parameter $\Psi$. The Euclidean-time action functional is given, in the natural units of $\hbar=1,2 m=1$, and $E_{F}=1$, by

$$
S(\beta)=\int_{0}^{\beta} d \tau \int d \boldsymbol{r}\left[\frac{D}{2}\left(\bar{\Psi} \frac{\partial \Psi}{\partial \tau}-\frac{\partial \bar{\Psi}}{\partial \tau} \Psi\right)+\mathcal{H}\right],
$$

where $\beta$ is the inverse temperature and the Hamiltonian $\mathcal{H}$ is given by

$$
\mathcal{H}=\Omega_{s}(\Psi)+\frac{C}{2 m}\left|\nabla_{r} \Psi\right|^{2}-\frac{E}{2 m}\left(\nabla_{r}|\Psi|^{2}\right)^{2} .
$$

The analytic expressions for the coefficients $C, D$, and $E$ of the EFT and for the thermodynamic potential $\Omega_{s}$ are given in $[26,30]$ in terms of the order parameter $\Psi$, chemical potential $\mu$, imbalance parameter $\zeta$, and interaction parameter $\left(k_{F} a_{s}\right)^{-1}$. To make this paper self-contained, the definitions of the EFT coefficients and a brief discussion about their relation to the system parameters are included in the Appendix. The regularized real-time Lagrangian density is

$$
\mathcal{L}=i \frac{D}{2}\left(\bar{\Psi} \frac{\partial \Psi}{\partial t}-\frac{\partial \bar{\Psi}}{\partial t} \Psi\right)-\left[\mathcal{H}-\Omega_{s}\left(\Psi_{\infty}\right)\right],
$$

where $\Psi_{\infty}$ is the value of the order parameter for a uniform system that can be obtained, given the values for temperature, interaction strength, and imbalance, by solving the gap equation $\partial \Omega_{s}(\Psi) / \partial \Psi=0$. The subtraction of the term $\Omega_{s}\left(\Psi_{\infty}\right)$ means that in the present treatment the energy is always intended as the energy difference with respect to the value of the thermodynamic potential for the uniform system.

From the effective-field Lagrangian, the equation of motion for the pair field $\Psi$ of the Fermi superfluid can be obtained:

$$
i \tilde{D}\left(|\Psi|^{2}\right) \frac{\partial \Psi}{\partial t}=-\frac{C}{2 m} \nabla_{r}^{2} \Psi+\left(A\left(|\Psi|^{2}\right)+\frac{E}{m} \nabla_{r}^{2}|\Psi|^{2}\right) \Psi .
$$

The coefficients $A$ and $\tilde{D}$ introduced in expression (4) are defined in (A8). In the 1D case, Eq. (4) can be solved analytically and an exact solution $\Psi_{s}\left(x-v_{s} t\right)$ for a stable soliton that propagates with velocity $v_{s}$ can be found. The properties of such solitonic solutions have been thoroughly examined in [30,31]. Moreover, in Ref. [30], the validity domain of the EFT was investigated and identified as the region of the $\left\{\left(k_{F} a_{s}\right)^{-1}, \beta, \zeta\right\}$ space where the pair field varies over a spatial region much larger than the pair correlation length [28,29]. This last quantity can be calculated in the EFT framework by using the method of the generating functional [36]. The 1D treatment, however, does not capture the physics of the snaking mechanism because, in order for the instability to develop, a transverse direction is needed. To describe the deformation of the soliton plane that leads to its decay, a transverse perturbation is added to the stationary 1D soliton in the following way [37]:

$$
\Psi(x, z, t)=\Psi_{s}\left(x-v_{s} t\right)+\Phi\left(x-v_{s} t, z, t\right),
$$

where the perturbation $\Phi\left(x-v_{s} t, z, t\right)$ is assumed to be small. The space and time dependence of the correction is assumed to have the form $x-v_{s} t$, meaning that it propagates in the $x$ direction with velocity $v_{s}$ in the same way that the soliton does. The perturbation is further assumed to consist of a combination of plane-wave components propagating in opposite directions:

$$
\Phi\left(x-v_{s} t, z, t\right)=\phi_{1}\left(x-v_{s} t\right) e^{i(k z-\Omega t)}+\phi_{2}^{*}\left(x-v_{s} t\right) e^{-i\left(k z-\Omega^{*} t\right)} .
$$

The next step is to insert this perturbed solution into the equation of motion (4) and to perform an expansion around the stationary solution up to first order in $\Phi$. From previous considerations [31], we know that the coefficients $C$ and $E$ can be kept constant and equal to their value in the uniform system case. On the other hand, the dependence of both $\tilde{D}$ and $A$ on the order parameter has to be fully considered. A Taylor expansion of these two coefficients up to first order around the stationary solution leads to

$$
\begin{aligned}
\tilde{D}\left(|\Psi|^{2}\right)= & \tilde{D}\left(\left|\Psi_{s}\right|^{2}\right)+\frac{\partial \tilde{D}\left(\left|\Psi_{s}\right|^{2}\right)}{\partial\left|\Psi_{s}\right|^{2}}\left[\left(\Psi_{s}^{*} \phi_{1}+\Psi_{s} \phi_{2}^{*}\right) e^{i(k z-\Omega t)}\right. \\
& \left.+\left(\Psi_{s} \phi_{1}^{*}+\Psi_{s}^{*} \phi_{2}\right) e^{-i(k z-\Omega t)}\right]+\cdots \\
A\left(|\Psi|^{2}\right)= & A\left(\left|\Psi_{s}\right|^{2}\right)+\frac{\partial A\left(\left|\Psi_{s}\right|^{2}\right)}{\partial\left|\Psi_{s}\right|^{2}}\left[\left(\Psi_{s}^{*} \phi_{1}+\Psi_{s} \phi_{2}^{*}\right) e^{i(k z-\Omega t)}\right. \\
& \left.+\left(\Psi_{s} \phi_{1}^{*}+\Psi_{s}^{*} \phi_{2}\right) e^{-i(k z-\Omega t)}\right]+\cdots
\end{aligned}
$$

After inserting (7) and (8) into the equation of motion and expanding the temporal and spatial derivatives, the terms of order zero in the perturbation can be collected, yielding

$$
i \tilde{D}\left(\left|\Psi_{s}\right|^{2}\right) \frac{\partial \Psi_{s}}{\partial t}=-\frac{C}{2 m} \nabla_{\boldsymbol{r}}^{2} \Psi_{s}+\left(A\left(\left|\Psi_{s}\right|^{2}\right)+\frac{E}{m} \nabla_{\boldsymbol{r}}^{2}\left|\Psi_{s}\right|^{2}\right) \Psi_{s},
$$

which is, as expected, just the equation of motion for the stationary solution. From the selection of the terms that are 
linear in the perturbation, two coupled differential equations are obtained for the perturbation amplitudes $\phi_{1}$ and $\phi_{2}$ :

$$
\begin{gathered}
\alpha_{1} \frac{\partial^{2} \phi_{1}}{\partial x^{2}}-\alpha_{2} \frac{\partial \phi_{1}}{\partial x}+\alpha_{3}(\Omega) \phi_{1}-\alpha_{4} \frac{\partial^{2} \phi_{2}}{\partial x^{2}}-\alpha_{5} \frac{\partial \phi_{2}}{\partial x}-\alpha_{6} \phi_{2}=0, \\
\alpha_{1} \frac{\partial^{2} \phi_{2}}{\partial x^{2}}-\alpha_{2}^{*} \frac{\partial \phi_{2}}{\partial x}+\alpha_{3}^{*}(-\Omega) \phi_{2}-\alpha_{4}^{*} \frac{\partial^{2} \phi_{1}}{\partial x^{2}}-\alpha_{5}^{*} \frac{\partial \phi_{1}}{\partial x}-\alpha_{6}^{*} \phi_{1}=0,
\end{gathered}
$$

where the coefficients $\alpha_{j}, j=1,2,3,4,5,6$, are defined as

$$
\begin{aligned}
\alpha_{1}= & \frac{C}{2 m}-\frac{E}{m}\left|\Psi_{s}\right|^{2}, \quad \alpha_{2}=i v_{s} \tilde{D}_{s}+2 \frac{E}{m} \Psi_{s} \frac{\partial \Psi_{s}^{*}}{\partial x}, \\
\alpha_{3}= & \Omega \tilde{D}_{s}-\frac{C}{2 m} k^{2}-\partial_{s}\left(\left|\Psi_{s}\right|^{2} A_{s}\right)-i v_{s} \partial_{s} \tilde{D}_{s} \frac{\partial \Psi_{s}}{\partial x} \Psi_{s}^{*} \\
& -\frac{E}{m} \frac{\partial^{2}\left|\Psi_{s}\right|^{2}}{\partial x^{2}}-\frac{E}{m} \Psi_{s} \frac{\partial^{2} \Psi_{s}^{*}}{\partial x^{2}}+\frac{E}{m}\left|\Psi_{s}\right|^{2} k^{2}, \\
\alpha_{4}= & \frac{E}{m} \Psi_{s}^{2} \frac{\partial^{2} \phi_{2}}{\partial x^{2}}, \quad \alpha_{5}=2 \frac{E}{m} \Psi_{s} \frac{\partial \Psi_{s}}{\partial x} \frac{\partial \phi_{2}}{\partial x}, \\
\alpha_{6}= & \partial_{s} A_{s} \Psi_{s}^{2}+i v_{s} \partial_{s} \tilde{D}_{s} \frac{\partial \Psi_{s}}{\partial x} \Psi_{s}+\frac{E}{m} \Psi_{s} \frac{\partial^{2} \Psi_{s}}{\partial x^{2}}-\frac{E}{m} \Psi_{s}^{2} k^{2} .
\end{aligned}
$$

In the last set of expressions we introduced the notation

$$
F\left(\left|\Psi_{s}\right|^{2}\right)=F_{s}, \quad \frac{\partial F}{\partial\left|\Psi_{s}\right|^{2}}=\partial_{s} F
$$

(where $F$ can stand for $A$ or $\tilde{D}$ ).

\section{RESULTS}

From the system of coupled differential equations (10) and (11) one can obtain information about the perturbation's frequency spectrum $\Omega(k)$. In particular, the soliton solution will be unstable for every wave vector $k$ that corresponds to an imaginary value of the frequency. Therefore, the first goal of the present work is to analyze the imaginary part of the spectrum $\Omega(k)$ and obtain a description of the growth rate of the instability in different interaction regimes across the BEC-BCS crossover. To do this, the system of equations is approached as an eigenvalue problem of the form

$$
\left(\begin{array}{ll}
W_{11} & W_{12} \\
W_{21} & W_{22}
\end{array}\right)\left(\begin{array}{l}
\phi_{1} \\
\phi_{2}
\end{array}\right)=\Omega\left(\begin{array}{l}
\phi_{1} \\
\phi_{2}
\end{array}\right)
$$

and is solved numerically for the case of a stationary soliton $\left(v_{S}=0\right)$ by approximating the derivatives with finite differences on a large space grid [38].

Figure 1 shows the results for the imaginary part of the eigenvalues $\Omega(k)$ at $T=0.01 T_{F}$ and for different values of the interaction parameter $\left(k_{F} a_{s}\right)^{-1}$. It is clear that the snake instability is a long-wavelength phenomenon that only exists up to a maximum wave number $k_{c}$ since the imaginary part of the frequency $\Omega$ is zero for $k>k_{c}$. The solid red line interpolates between the values of $\operatorname{Im}[\Omega(k)]$ calculated in $k=k_{c} / \sqrt{2}$. As predicted by Muryshev et al. in the case of Bose-Einstein condensates [15], this line nicely connects the maxima of the dispersion relations for different $\left(k_{F} a_{s}\right)^{-1}$.

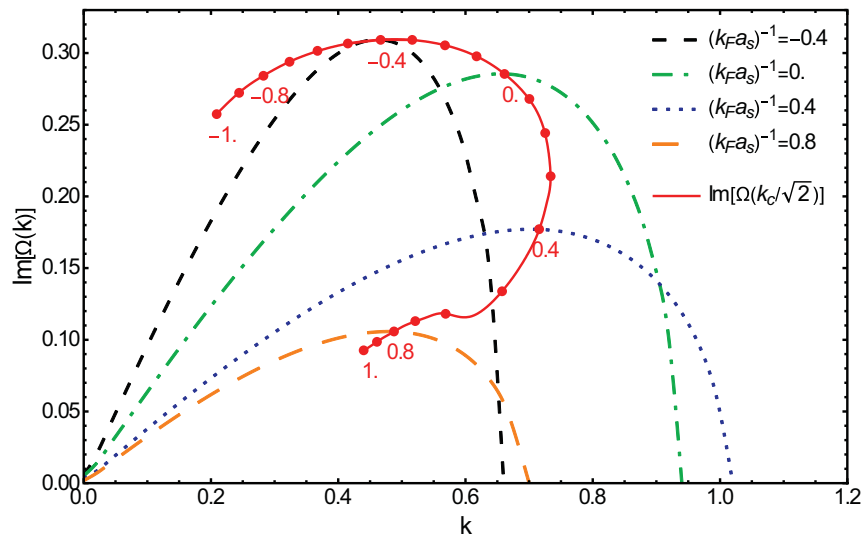

FIG. 1. Dispersion relations for the snake instability in different interaction conditions across the BEC-BCS crossover, i.e., on the BCS side of the resonance at $\left(k_{F} a_{s}\right)^{-1}=-0.4$ (black dashed line), at unitarity $\left(k_{F} a_{s}\right)^{-1}=0$ (green dash-dotted line), in the near-BEC regime $\left(k_{F} a_{s}\right)^{-1}=0.4$ (blue dotted line), and further towards the BEC limit at $\left(k_{F} a_{s}\right)^{-1}=0.8$ (orange long-dashed line). The solid red line connects the values of $\operatorname{Im}[\Omega(k)]$ calculated in $k=k_{c} / \sqrt{2}$ for different values of $\left(k_{F} a_{s}\right)^{-1}$. The markers correspond to values of $\left(k_{F} a_{s}\right)^{-1}$ ranging from -1 to 1 in steps of 0.1 . The wave number $k$ is given in units of $k_{F}$ and the frequency $\operatorname{Im}[\Omega]$ in units of $\Omega_{F}=E_{F} / \hbar$.

Figure 2 compares the results for $\left(k_{F} a_{s}\right)^{-1}=0$ (left panel) and $\left(k_{F} a_{s}\right)^{-1}=0.2$ (right panel) with the corresponding spectra that were calculated in Ref. [23]. There, the authors made use of three different approaches to analyze the spectra of the snake instability: a hydrodynamic approximation, the RPA, and the solution of the time-dependent Bogoliubov-de Gennes (TDBdG) equations. As concerns the width of the band of unstable wavelengths, the latter method shows the best agreement with the EFT results. The RPA results, on the other hand, show a sharp decrease of $\operatorname{Im}[\Omega]$, which might be caused by the necessary use of an energy cutoff in this type of calculation, an issue that does not occur in the presently used EFT. Another consequence of this cutoff is that the RPA method fails to find any imaginary frequency at all for $\left(k_{F} a_{s}\right)^{-1}>0.2$. The hydrodynamic approximation, which describes a linear relation between $\Omega$ and $k$, is only expected to hold near $k=0$, where it indeed agrees quite well with the initial slope of the present results.

The existence of a minimum wave number $k_{c}$ for which $\operatorname{Im}[\Omega]$ becomes zero implies that there exists a minimal transverse length the ultracold gas must have in order for the soliton to decay. If the transverse width is smaller than this minimal value, the soliton is expected to be stable. A good estimate for this critical length is given by the inverse of $k_{c}$. In Fig. 3 this quantity is compared to the RPA and TDBdG results of [23] as well as to the data from [24] relative to a treatment based on the coarse-grained BdG equation introduced by Simonucci and Strinati [25]. Numerical factors have been introduced after a cross comparison between Refs. [15,23,24] in order to overcome the difference in the definitions of the healing lengths [39]. The EFT values (black solid line) appear to be in good agreement with the TDBdG results (blue circles with error bars) across the whole range of available data. Moreover, it seems that the present EFT approach captures 

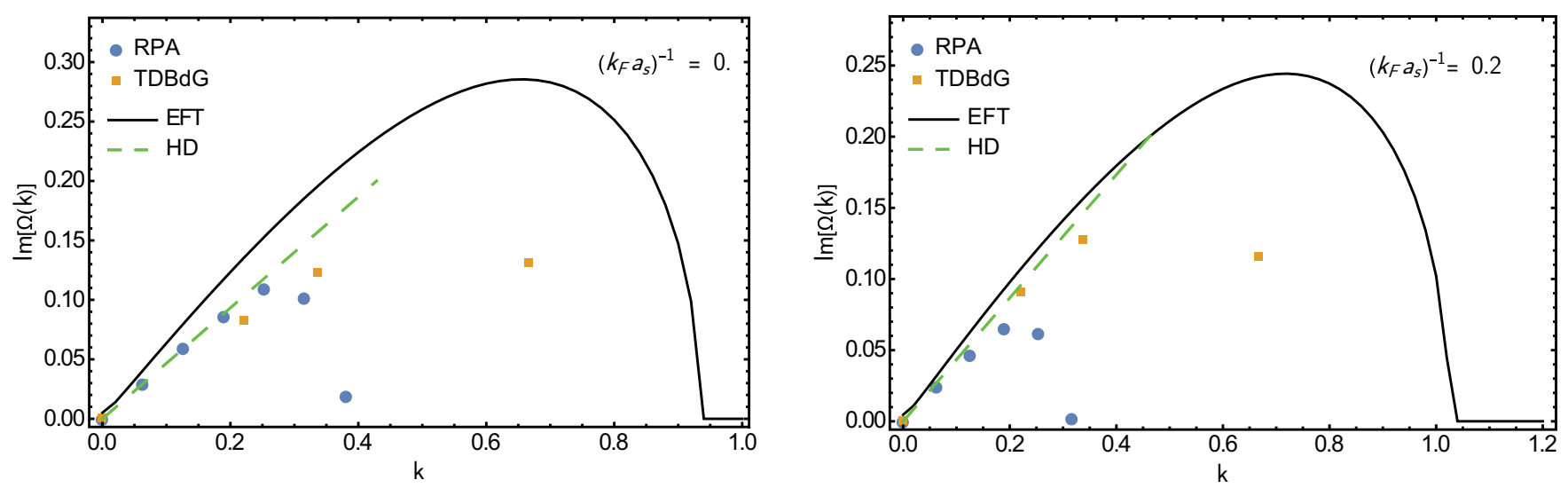

FIG. 2. Dispersion relations for the snake instability for $\left(k_{F} a_{s}\right)^{-1}=0$ (left panel) and $\left(k_{F} a_{s}\right)^{-1}=0.2$ (right panel). The solid black line represents the EFT prediction and it is compared to the results of the hydrodynamic approximation (green dashed line), of the RPA (blue circles), and of the TDBdG simulations (orange squares) [23]. The wave number $k$ is given in units of $k_{F}$ and the frequency $\operatorname{Im}[\Omega]$ in units of $\Omega_{F}$.

well the fact that the characteristic length of the system changes from the healing length in the BEC regime (purple dash-double-dotted line) to the correlation length in the BCS regime (green dashed line). In the far BEC limit the EFT results are in excellent agreement with both the data from [24] (red dash-dotted line) and with the healing length obtained from the standard Gross-Pitaevskii treatment, which can therefore be identified as the relevant length scale for the decay process.

It should be observed that, while the discussion carried out in [30] about the validity domain of the EFT reveals that the reliability of the EFT results on the BCS side of the resonance is not guaranteed at low temperatures, the comparison with the benchmark TDBdG results in said regime confirms the validity

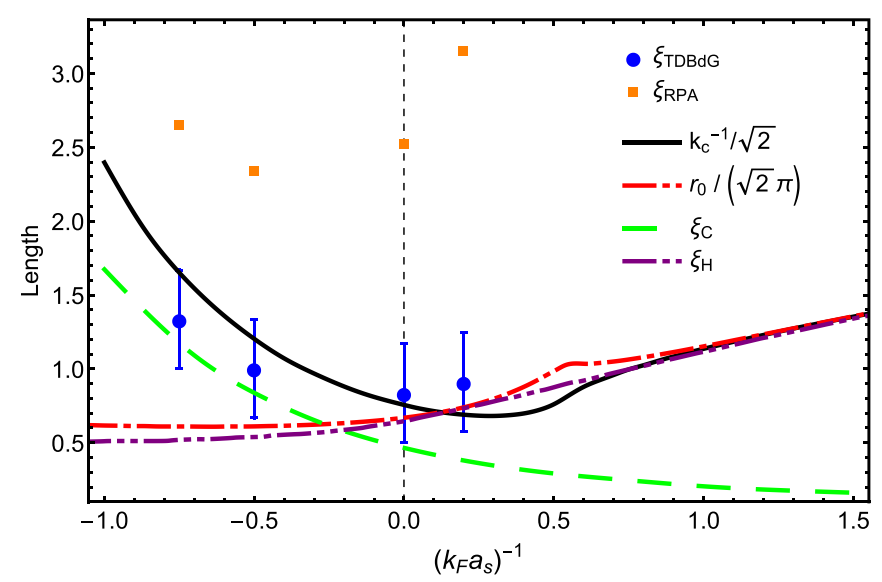

FIG. 3. The EFT prediction for the minimum transverse dimension necessary for observing soliton decay through the snake instability (solid black line) is compared to the results of the RPA (orange squares), of the TDBdG simulations (blue circles) [23], and of the calculations by Muñoz Mateo and Brand [24] (red dash-dotted line) based on the coarse-grained BdG theory [25]. In addition, the BCS coherence length (green dashed line) and BEC healing length (purple dash-double-dotted line) are shown. The numerical factors are introduced to overcome differences in the definitions of the healing lengths between Refs. [15,23,24] as discussed in [39]. The length is given in units of $k_{F}^{-1}$. of the present calculations. This can be explained by the fact that the snake instability is an intrinsically long-wavelength phenomenon and therefore complies well with the requirement of a slowly varying order parameter.

In Fig. 4 the effect of spin imbalance on the critical wave number for the instability is examined. It appears that the presence of unpaired particles stabilizes the soliton: The value of $k_{c}$ at a fixed interaction strength decreases when increasing the imbalance parameter $\zeta$, meaning that for a given width of the atomic cloud a soliton in an imbalanced setup can be stable, while one in a balanced system is unstable. This behavior has recently been confirmed by TDBdG calculations [40]. This can be qualitatively explained in terms of the observation that in an imbalanced configuration the soliton core is an energetically

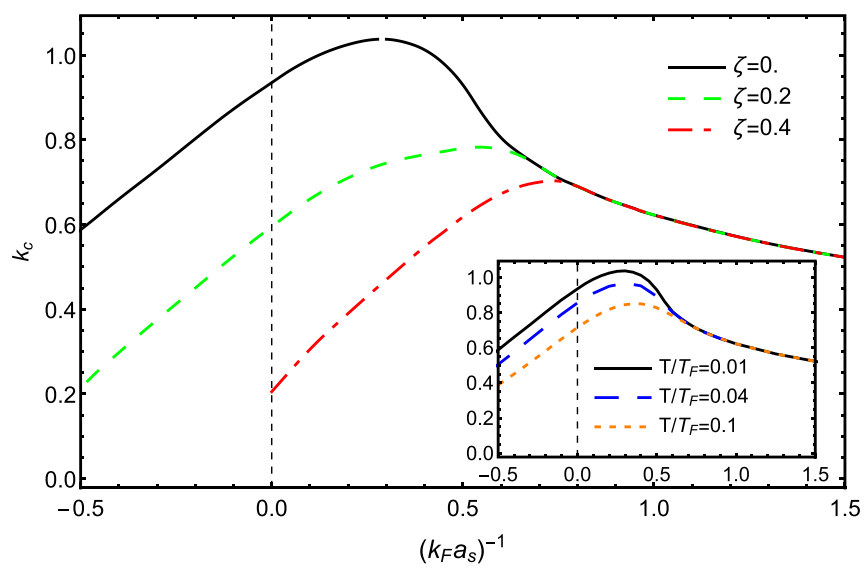

FIG. 4. Critical wave number $k_{c}$ for the occurrence of the snake instability across the BEC-BCS crossover for different values of the imbalance parameter $\zeta$, i.e., $\zeta=0$ (solid black line), $\zeta=0.2$ (green dashed line), and $\zeta=0.4$ (red dash-dotted line). The lines for $\zeta \neq 0$ do not cover the entire interaction domain due to the fact that in the presence of imbalance the superfluid state does not exist across the whole BEC-BCS crossover. The inset shows the behavior of the same quantity at finite temperature, i.e., $T=0.01 T_{F}$ (solid black line), $T=0.04 T_{F}$ (blue dashed line), and $T=0.1 T_{F}$ (orange dash-dotted line). The critical wave number is given in units of $k_{F}$. 
favorable place to accommodate the unpaired particles [30]. Because of this the system may favor the soliton configuration over the vortex one since the former offers more space to store the excess component particles. Additional evidence in favor of this observation comes from the analysis of the finitetemperature behavior of $k_{c}$. As it is clear from the inset of Fig. 4, an increase in temperature, by enhancing the number of unpaired particles in the system, also produces a decrease of the critical value $k_{c}$.

\section{CONCLUSION}

In this paper the snake instability mechanism for dark solitons in Fermi superfluids was studied in different conditions of interaction and imbalance. The theoretical treatment is based on an effective-field theory [26] already employed to discuss the properties of stable dark solitons in quasi$1 \mathrm{D}$ configurations. The distortion of the depletion plane characteristic of the onset of the snake instability is treated by adding a transverse perturbation to the stable solution $\Psi_{s}$ of the equations of motion for the quasi-1D configuration in the form (6). The numerical solution of the coupled system of nonlinear differential equations describing the perturbation amplitude provides the spectra of the instability. The growth rate is examined in different interaction regimes and the BEC prediction [15] for the position of the maxima of $\operatorname{Im}[\Omega(k)]$ is verified and extended to the BCS side of the resonance. The minimal transverse size for the soliton decay is qualitatively estimated as $k_{c}^{-1}, k_{c}$ being the maximal wave number for which unstable modes exist. The results obtained show good quantitative agreement with those of the coarse-grained $\mathrm{BdG}$ theory [24] in the BEC regime and the available numerical results of the TDBdG calculations [23] across the crossover. Moreover, the EFT results seem to correctly characterize the change in the relevant length scale, from the condensate healing length in the BEC limit to the correlation length in the BCS regime.

At a later stage in the paper the effects of spin imbalance and temperature on the stability of the soliton are discussed. In particular, the maximum transverse size that the atomic cloud can have in order for the soliton to be stable is shown to increase in the presence of spin imbalance or at finite temperature, in agreement with the observation that it is energetically favorable for the additional unpaired particles to fill the soliton core $[30,40]$. This could in principle offer a method to stabilize the soliton configuration in experiments without being forced to reduce the transverse size of the trap. The analysis carried out in the present paper is based on the perturbation of stable solitons solutions derived in [30,31], which were obtained under the hypothesis of a uniform system. While most experiments concerning ultracold quantum gases employ harmonic traps to confine the atomic cloud, recently, boxlike optical traps that well approximate a uniform configuration were developed [32]: Such setups can provide the opportunity to test the predictions of this work in experiment.

\section{ACKNOWLEDGMENTS}

We gratefully acknowledge useful discussions with J. P. A. Devreese and N. Verhelst. W.V.A. gratefully acknowledges support in the form of a Ph.D. fellowship from the Research Foundation-Flanders. This research was supported by the Flemish Research Foundation, Projects No. G.0115.12N, No. G.0119.12N, No. G.0122.12N, No. G.0429.15N, and No. G0G6616N; the Scientific Research Network of the Research Foundation-Flanders, Grant No. WO.033.09N; and the Research Fund of the University of Antwerp.

\section{APPENDIX: COEFFICIENTS OF THE EFT}

In this appendix we give an overview of the coefficients appearing in the effective-field action (1). It is convenient to write these coefficients in terms of the functions $f_{j}(\beta, \epsilon, \zeta)$, which are defined as the solutions of

$$
f_{j}(\beta, \epsilon, \zeta)=\frac{1}{\beta} \sum_{n} \frac{1}{\left[\left(\omega_{n}-i \zeta\right)^{2}+\epsilon^{2}\right]^{j}},
$$

where $\omega_{n}$ are fermionic Matsubara frequencies of the form $\omega_{n}=(2 n+1) \pi / \beta$. The explicit expression for the first of these functions $f_{1}(\beta, \epsilon, \zeta)$ is given by

$$
f_{1}(\beta, \epsilon, \zeta)=\frac{1}{2 \epsilon} \frac{\sinh (\beta \epsilon)}{\cosh (\beta \epsilon)+\cosh (\beta \zeta)} .
$$

From this, the other $f_{j}(\beta, \epsilon, \zeta)$ with $j=2,3, \ldots$ can be calculated by using the simple recursion relation

$$
f_{j+1}(\beta, \epsilon, \zeta)=-\frac{1}{2 j \epsilon} \frac{\partial f_{j}(\beta, \epsilon, \zeta)}{\partial \epsilon} .
$$

The complete expressions for the coefficients appearing in $S(\beta)(1)$ are hence given by

$$
\begin{gathered}
C=\int \frac{d \mathbf{k}}{(2 \pi)^{3}} \frac{k^{2}}{3 m} f_{2}\left(\beta, E_{\mathbf{k}}, \zeta\right), \\
D=\int \frac{d \mathbf{k}}{(2 \pi)^{3}} \frac{\xi_{\mathbf{k}}}{w}\left[f_{1}\left(\beta, \xi_{\mathbf{k}}, \zeta\right)-f_{1}\left(\beta, E_{\mathbf{k}}, \zeta\right)\right], \\
E=2 \int \frac{d \mathbf{k}}{(2 \pi)^{3}} \frac{k^{2}}{3 m} \xi_{\mathbf{k}}^{2} f_{4}\left(\beta, E_{\mathbf{k}}, \zeta\right),
\end{gathered}
$$

while the thermodynamic potential $\Omega_{s}$ reads

$$
\begin{aligned}
\Omega_{s}(\Psi)= & -\int \frac{d \boldsymbol{k}}{(2 \pi)^{3}}\left[\frac{1}{\beta} \ln \left[2 \cosh \left(\beta E_{\boldsymbol{k}}\right)+2 \cosh (\beta \zeta)\right]\right. \\
& \left.-\xi_{\boldsymbol{k}}-\frac{m|\Psi|^{2}}{k^{2}}\right]-\frac{m|\Psi|^{2}}{4 \pi a_{s}} .
\end{aligned}
$$

The chemical potentials of the two pseudospin species $\mu_{\uparrow}$ and $\mu_{\downarrow}$ have been combined into the average chemical potential $\mu=\left(\mu_{\uparrow}+\mu_{\downarrow}\right) / 2$ and the imbalance chemical potential $\zeta=$ $\left(\mu_{\uparrow}-\mu_{\downarrow}\right) / 2$. This last parameter determines the difference between the number of particles in each spin population. The quantity $\xi_{k}=\frac{k^{2}}{2 m}-\mu$ is the dispersion relation for a free fermion, $E_{k}=\sqrt{\xi_{k}+|\Psi|^{2}}$ is the single-particle excitation energy, and $a_{s}$ is the $s$-wave scattering length that determines the strength and sign of the contact interaction. The coefficients $A$ and $\tilde{D}$ appearing in the equation of motion (4) are defined as

$$
A=\frac{\partial \Omega_{s}(\Psi)}{\partial|\Psi|^{2}}, \quad \tilde{D}=\frac{\partial\left(|\Psi|^{2} D\right)}{\partial|\Psi|^{2}} .
$$


[1] J. Denschlag, J. E. Simsarian, D. L. Feder, C. W. Clark, L. A. Collins, J. Cubizolles, L. Deng, E. W. Hagley, K. Helmerson, W. P. Reinhardt, S. L. Rolston, B. I. Schneider, and W. D. Phillips, Science 287, 97 (2000).

[2] S. Burger, K. Bongs, S. Dettmer, W. Ertmer, K. Sengstock, A. Sanpera, G. V. Shlyapnikov, and M. Lewenstein, Phys. Rev. Lett. 83, 5198 (1999).

[3] B. P. Anderson, P. C. Haljan, C. A. Regal, D. L. Feder, L. A. Collins, C. W. Clark, and E. A. Cornell, Phys. Rev. Lett. 86, 2926 (2001).

[4] C. Becker, S. Stellmer, P. Soltan-Panahi, S. Dörscher, M. Baumert, E.-M. Richter, J. Kronjger, K. Bongs, and K. Sengstock, Nat. Phys. 4, 496 (2008).

[5] T. Yefsah, A. T. Sommer, M. J. H. Ku, L. W. Cheuk, W. Ji, W. S. Bakr, and M. W. Zwierlein, Nature (London) 499, 426 (2013).

[6] M. J. H. Ku, W. Ji, B. Mukherjee, E. Guardado-Sanchez, L. W. Cheuk, T. Yefsah, and M. W. Zwierlein, Phys. Rev. Lett. 113, 065301 (2014).

[7] M. J. H. Ku, B. Mukherjee, T. Yefsah, and M. W. Zwierlein, Phys. Rev. Lett. 116, 045304 (2016).

[8] V. V. Konotop and L. Pitaevskii, Phys. Rev. Lett. 93, 240403 (2004).

[9] D. J. Frantzeskakis, J. Phys. A: Math. Theor. 43, 213001 (2010).

[10] M. Antezza, F. Dalfovo, L. P. Pitaevskii, and S. Stringari, Phys. Rev. A 76, 043610 (2007).

[11] A. Spuntarelli, P. Pieri, and G. Strinati, Phys. Rep. 488, 111 (2010).

[12] R. Liao and J. Brand, Phys. Rev. A 83, 041604 (2011).

[13] R. G. Scott, F. Dalfovo, L. P. Pitaevskii, and S. Stringari, Phys. Rev. Lett. 106, 185301 (2011).

[14] S. Dutta and E. J. Mueller, Phys. Rev. Lett. 118, 260402 (2017).

[15] A. E. Muryshev, H. B. van Linden van den Heuvell, and G. V. Shlyapnikov, Phys. Rev. A 60, R2665 (1999).

[16] D. L. Feder, M. S. Pindzola, L. A. Collins, B. I. Schneider, and C. W. Clark, Phys. Rev. A 62, 053606 (2000).

[17] J. Brand and W. P. Reinhardt, Phys. Rev. A 65, 043612 (2002).

[18] V. E. Zakharov and A. M. Rubenchik, Sov. Phys.-JETP 38, 494 (1974).

[19] S. Donadello, S. Serafini, M. Tylutki, L. P. Pitaevskii, F. Dalfovo, G. Lamporesi, and G. Ferrari, Phys. Rev. Lett. 113, 065302 (2014).
[20] M. Ma, R. Carretero-González, P. G. Kevrekidis, D. J. Frantzeskakis, and B. A. Malomed, Phys. Rev. A 82, 023621 (2010).

[21] S. K. Adhikari, Phys. Rev. A 89, 043615 (2014).

[22] S. Adhikari, Laser Phys. Lett. 13, 035502 (2016).

[23] A. Cetoli, J. Brand, R. G. Scott, F. Dalfovo, and L. P. Pitaevskii, Phys. Rev. A 88, 043639 (2013).

[24] A. Muñoz Mateo and J. Brand, Phys. Rev. Lett. 113, 255302 (2014).

[25] S. Simonucci and G. C. Strinati, Phys. Rev. B 89, 054511 (2014).

[26] S. N. Klimin, J. Tempere, G. Lombardi, and J. T. Devreese, Eur. Phys. J. B 88, 122 (2015).

[27] L. P. Gor'kov, Sov. Phys._JETP 36, 1364 (1959).

[28] F. Pistolesi and G. C. Strinati, Phys. Rev. B 49, 6356 (1994).

[29] F. Palestini and G. C. Strinati, Phys. Rev. B 89, 224508 (2014).

[30] G. Lombardi, W. Van Alphen, S. N. Klimin, and J. Tempere, Phys. Rev. A 93, 013614 (2016).

[31] S. N. Klimin, J. Tempere, and J. T. Devreese, Phys. Rev. A 90, 053613 (2014).

[32] A. L. Gaunt, T. F. Schmidutz, I. Gotlibovych, R. P. Smith, and Z. Hadzibabic, Phys. Rev. Lett. 110, 200406 (2013).

[33] M. W. Zwierlein, A. Schirotzek, C. H. Schunck, and W. Ketterle, Science 311, 492 (2006).

[34] G. B. Partridge, W. Li, R. I. Kamar, Y.-A. Liao, and R. G. Hulet, Science 311, 503 (2006).

[35] J. Tempere, S. N. Klimin, and J. T. Devreese, Phys. Rev. A 79, 053637 (2009).

[36] G. Lombardi, Effective field theory for superfluid Fermi gases, Ph.D. thesis, Universiteit Antwerpen, 2017.

[37] E. A. Kuznetsov and S. K. Turitsyn, Sov. Phys._JETP 67, 1583 (1988)

[38] J. T. Cole and Z. H. Musslimani, Physica D 313, 26 (2015).

[39] In [24] the quantity $r_{0}$ is defined as $r_{0}=(\pi / \sqrt{-2 \lambda}) \xi$. Therefore, the data plotted in Fig. 3, i.e., $r_{0} / \sqrt{2} \pi$, describe a corrected healing length accounting for the modulation effect due to the variation of the (bound) ground-state eigenvalue $\lambda$ across the BEC-BCS crossover. The factor $1 / \sqrt{2}$ comes instead from a difference in the definition of $\xi$ with respect to [15] .

[40] M. D. Reichl and E. J. Mueller, Phys. Rev. A 95, 053637 (2017). 